\title{
RADICULOPATIA NEOPLÁSICA LOMBOSSACRAL
}

\author{
SANDRO L. ROSSITTI* - ANTONIO A. ROTH-VARGAS** \\ ALEXANDER SPERLESCU*** ROQUE J. BALBO****
}

\begin{abstract}
RESUMO - Comentárfo crítico da rotina radiodiagnóstica nas radiculopatias lombossacrais no que concerne ao uso da tomografia computadorizada (C'T). São relatadas as observaçōes clinicas de três pacientes nos quais neoplasias acometeram una única raiz lombossacral, simulando uma hérnia de disco lombar do ponto de vista clínico, com ênfase nos aspectos neurorradiológlcos. Reconjenda-se o estudo sistemático do sacro e da zona do cone medular em todo caso de radiculopatia lombossiacral.
\end{abstract}

\section{Neoplasic lumbosacral radiculopathy.}

SUMMARY - Lumbar-disc protrusions (LDP) constitute well-defined syndromes on clinical and anatomical grounds, and neurosurgeons are prone to rely upon the clinical signs to identify the level of disc protrusion when a atypical» case is found. Sometimes, non-contrasted computerized tomographic (CT) scans centered on the I5-S1, LA-L5 and IB-LA interspaces and spine roentgenograms are the only special ancilary means in presurgical evaluation. We report three patients from our series, in which neoplasic spinal disease presented as classic LDP (one patient with a cauda equina schwannoma, and two with metastatic carcinoma). The cases were chosen because they posed special problems to the referred radiodiagnostic routine. Systematic Cr-evaluation of the sacrum and conus medullaris zone is recommended in every patient with lumbosacral radiculopathy, and intratecal contrast should be employed in patients with unreliable findings or normal CT-scans.

As radiculopatias lombossacrais por herniaçăo posterior do núcleo pulposo constituem sindromes bem caracterizadas do ponto de vista anátomo-clínico 4 . Muitos pacientes, porém, apresentam dor radicular de outra natureza. Qualquer lesão que cause compressão radicular, em especial quando uma única raiz é envolvida, pode ser facilmente confundida com uma hérnia de disco intervertebral (HNP); entre essas lesōes, citamos: espondilite tuberculosa ou piogênica, espondilite anquilosante, fraturas, neoplasias espinais, schwannoma do nervo ciático, estenose do canal raqueano, aracnoidite, neuropatias paraneoplásicas e diabéticas; o diagnóstico diferencial deve levar em consideração também estados histéricos e neoplasias do pâncreas e da pelve; em nosso meio devemos lembrar ainda a neurocisticercose $2,5,6$. Certas condições desencadeantes, como o trauma e o esforço muscular, referidos em cerca de 50\% das HNP, podem também iniciar a história clínica de neoplasias e de processos inflamatórios 2,5. Uma história clínica e exame físico minuciosos e exames radiológicos de rotina podem afastar muitas dessas possíveis causas.

A propedêutica neurorradiológica tem por objetivo confirmar a localização clinica e determinar se mais de uma HNP se faz presente; contribui também no diag-

Departamento de Neuro-Psiquiatria da Faculdade de Ciências Médicas da Pontifícia Universidade Católica de Campinas (PUCCAMP) e Departamento de Neurocirurgia do Hospital Vera Cruz (HVC), Campinas: * Médico Residente; ** Neurocirurgiāo do HVC; *** Professor Assistente; **** Professor Adjunto, Diretor do Departamento de Neurocirurgia do HVC. 
nóstico diferencial com outras causas de lombociatalgia. Elaboramos a seguir comentário crítico da rotina radiodiagnóstica nas radiculopatias lombossacrais, em especial no que concerne ao emprego cada vez mais generalizado da tomografia computadorizada (CT) da coluna como único auxílio diagnóstico especial, ilustrado por alguns casos clinicos de nossa experiência recente, escolhidos por trazerem problemas altamente significativos para os comentários que se seguem.

\section{OBSERVAÇÕES}

Crso 1 - JGRB, paciente do sexo masculino, com 31 anos de idade, encraminhado a nossa Clínica em abril de 1987 com ciatalgia direita há 7 meses. Radiografias simples $\Theta$ CT da coluna normais (Fig. 1). Eletroneuromiografia (ENMG) revelou radiculopatia crônica S1 direita. Indicamos tratamento conservador. Como reagudizassem os sintomas apos 4 meses, realizamos mielografia lombar que revelou legão intradural no nível LA-L5 (Fig. 2), a qual após extirpacāo cirúrgica verificamos tratar-se de um neurinoma. o paciente teve boa evoluçăo, encontrando-se assintomático após 22 meses.
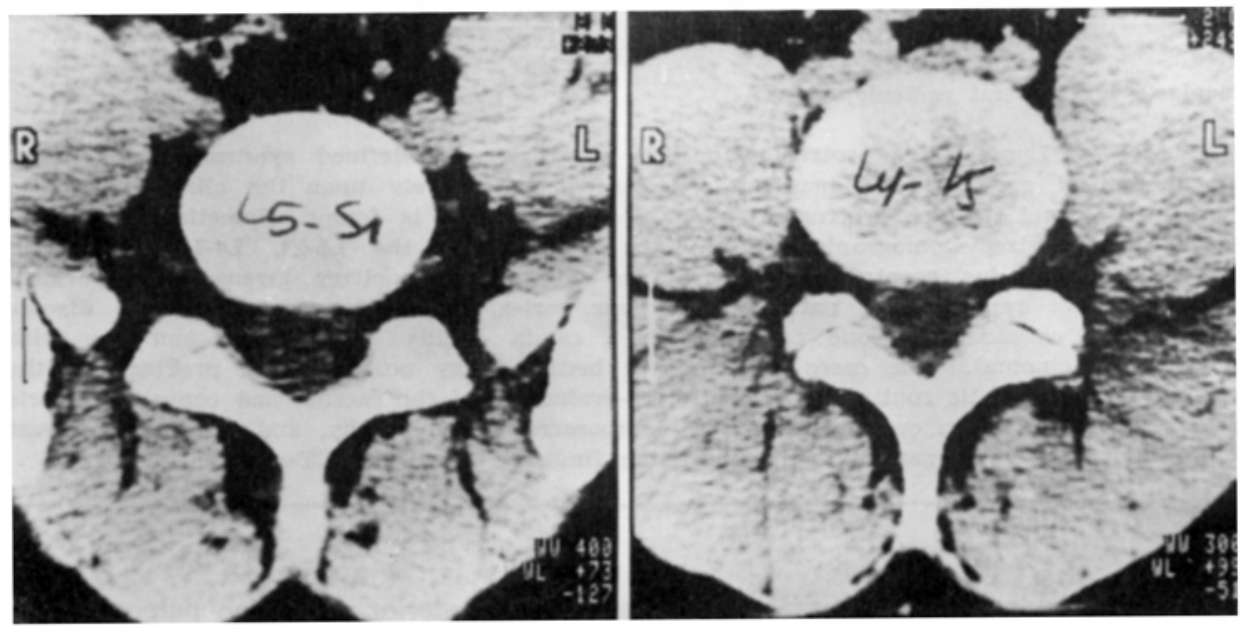

Fig. 1 - Caso JGRB. CT da coluna vertebral ao nivel do interespaco L5-\$1 (em A, à esquerda) $e$ ao nivel de L4-L5 (em $B$, à direita). O exame foi considerado normal.

C'uso 2 - CL, paciente do sexo feminino, com 58 anos de idade, onerada há dois anos e meio de adenocarcinoma do reto (amputacão do reto e colostomia), tendo recebido radiaterapia complemertar. Há 6 meses apresenta lombociatalgia esquerda. Radiografias do tórax. abdome e ossos da pelve normais. Radiografias da coluna vertebral revelaram sinais de espondiloartrase difusa leve. CT da pelve e abdome não acusou sinais de recidiva tumoral. Encaminhado a nossa Clinica com hipótese diagnóstica de HNP em setembro de 1987 . CT da coluna revelou espessamento da raiz $\mathbf{S} 1$ esquerda em seu forame de emergência sacral (Fig. 3). Exploração cirúrgica revelou tratar-se de infiltração neoplásica por adenocarcinoma poucto diferenciado; realizamos rizotomia posterior da raiz infiltrada para controle ds dor. Recidiva tumoral, com infiltracão do plexo lombossacral esquerdo, sendo necessária cordotomia anterolateral (nivel Th) em ianeiro de 1989, para controle da dor.

Caso 3 - AAD, paciente do sexo feminino, com 31 anos de idade, operada há 5 anos de carcinoma da bexiga, sendo considerada a remoçăo cirúrgica radical; foi indicada radioterapia complenentar, que a paciente recusou. Permaneceu assintomática desde entåo. Encaminhada a nossa Clinica em dezembro de 1987, com queixa de lombociatalgia direita há 7 meses, de início súbito após queda da própria altura. Radiografias do tórax, ossos da bacia e coluna vertebral consideradas normais. CT da coluna e pelve revelou infiltração sacral à direita por massa tumoral pelvica (Fig. 3). Abordagem cirúrgica por via posterior 


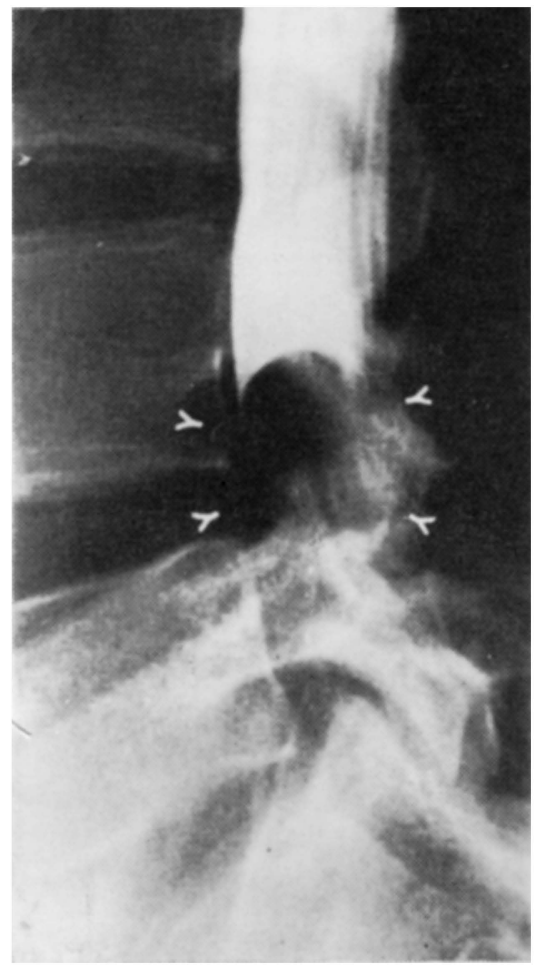

Fig. 2 - Caso JGRB. Mielografia lombar. Presença de lesão expansiva intradural, com bloqueio da coluna de contraste ao nivel do corpo vertebral de $L_{4}$ (Schwannoma).

revelou raiz nervosa infiltrada por neoplasia; realizamos rizotomia posterior para controle da dor. Estudo histológico revelou carcinoma pouco diferenciado. A paciente foi encaminhada a Serviço de Kadioterapia após consulta com o urologista que a operou inicialmente.

Em todos esses pacientes encontramos sinais irritativos (dor lombar irradiada até a borda externa do pé, parestesias na área de irradiação dolorosa, contratura antáleica da musculatura paravertebral lombar, limitach̆o dos movimentos espinhais e presenca do sinal de Lasègue) a compressivos (hipoestesia superficial leve na região lateral e posterior da perna e borda lateral do pé, fraqueza da musculatura da panturrilha e pequenos músculos do pé, inibição do reflexo aquileu), estes últimos mais pronunciados no caso 3 , sugestivos de uma radiculopatia s1. Todos encontravam-se em excelente estado geral e nas duas pacientes com neoplasia maligna (casos 2 e 3) não encontramos metástases à distáncia; nesses casos o relato de doença maligna pregressa tornou suspelta a natureza da lesăo, mas a história clínica recente e o exame neurológico impuseram o diagnóstico diferencial com HNP. As alteracões 6sseas reveladas em radiografias simples resumiram-se a espondiloartrose em uma única paciente (caso 2); em nenhum caso observou-se lesăo osteolítica. A C' $\Gamma$ mostrou-se normal ao nivel dos interespacos discais lombares em todos os casos, localizando-se a lesão em nivel intrassacral em duas pacientes (casos 2 e 3), e intradural lombar em outro (caso 1). eate revelado adenas com o emoregn de contraste intratecal.

\section{COMENTARIOS}

As síndromes de herniação posterior do núcleo pulposo na região lombossacral estão bem caracterizadas de longa data, na maioria dos casos sendo possivel diagnóstico preciso com base tão somente na anamnese e exame clínico 2,4. Sendo essa condição muito freqüente, temos tendência a atribuir uma discopatia a todo paciente com lombociatalgia, com prejuízo para o diagnóstico de outras causas de radiculopatia quando um caso "típico" é encontrado. 

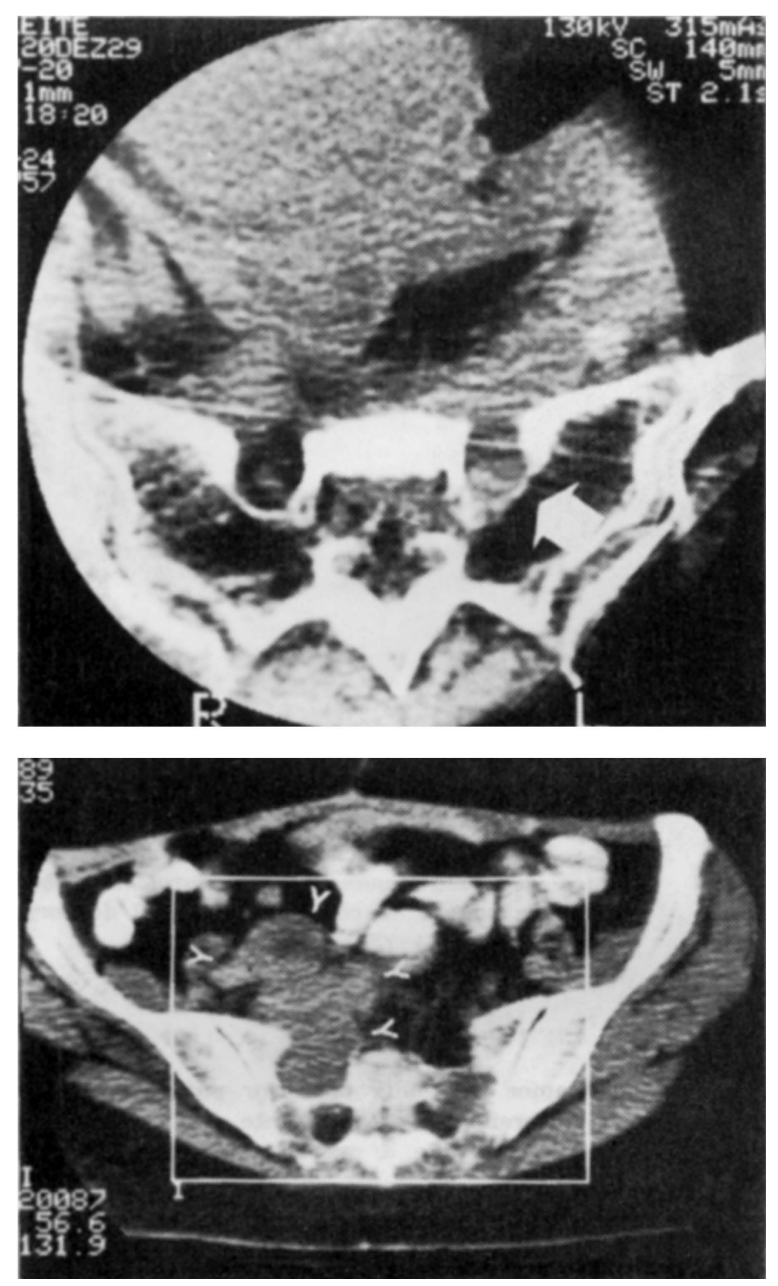

Fig. 3 - Em cima, caso CL: CT da coluna vertebral, corte ao nivel intrassacral; note-se espessamento da raiz $\mathrm{S1}$ esquerda (infiltração carcinomatosa). Em baixo, caso $A A D: C T$ da coluna vertebral $e$ pelve; processo tumoral pélvico infiltrando o sacro à direita, no forame de emergência da raiz $\mathrm{S} 1$ (infiltração carcinomatosa).

A CT da coluna vertebral oferece boa precisão diagnóstica nos casos de HNP lombar, fornecendo imagens bem definidas (porém de extensão restrita) do disco intervertebral, da disposição anatômica das raízes e do saco dural, da gordura peridural; é também bastante conclusiva na estenose do canal raqueano e nas artropatias facetárias 3. Considerada "procedimento năo-invasivo", tem sido cada vez mais empregada na última década por muitos neurocirurgióes como o primeiro, senăo o único, exame complementar diagnóstico especial nas lombociatalgias. Portadores de sintomas radiculares lombossacrais são, em geral, sistematicamente estudados quanto às discopatias dessa região, nos interespaços L5-S1, L4-L5 e L3-L4. Permanece aí uma lacuna no estudo do trajeto das raizes na região intrassacral e na zona do cone medular (L1-L2). Uma lesão intradural pode năo trazer qualquer compromisso ósseo, passando desapercebida em radiografias simples e ao CT sem contraste intratecal (caso 1). Há ainda o risco de indicarmos cirurgia sobre uma protrusão discal "inocente" no segmento vertebral estudado, quando a lesão se encontra em outro local. Problema semelhante ocorre nas mielografias em que a coluna de contraste não for devidamente levada à zona do cone medular. Nos últimos anos, as imagens obtidas por ressonância magnética nuclear (NMR) revolucionaram o diagnóstico das doenças espinhais 1. A NMR tem se mostrado particularmente eficaz no estudo das malformaçōes congênitas, tumores e infecções espinhais. Todavia, no atual estado 
da tecnologia de NMR, a CT oferece acuidade diagnóstica igual ou superior nas degeneraçōes do disco intervertebral e no traumatismo raque-medular agudo.

Concluimos, assim, pela análise dos casos apresentados (nos quais neoplasias acometeram uma única raiz nervosa, simulando uma HNP do ponto de vista clínico), que a referida rotina radiodiagnóstica é insuficiente. Recomendamos que sejam estudadas sistematicamente a região intrassacral e a zona do cone medular em todo paciente com monorradiculopatia lombossacral, reservando a mielografia para os casos em que os achados tomográficos forem normais ou insuficientes 5 . Quanto à NMR, trata-se de técnica em rápida evolução e aguardamos sua maior disponibilidade para situá-la em seu legitimo lugar na rotina radiodiagnóstica.

\section{REFERENCIAS}

1. Dernbach PD, Weinstein MA, Little JR - Magnetic resonance imaging of spinal disorders. Clin Neurosurg $\mathbf{3 4 : 2 6 1 , 1 9 8 8 .}$

2. Love JG - The differential diagnosis of intraspinal tumors and protruted intervertebral disks and their surgical treatment. J Neurosurg 1:275, 1944.

3. Meyer GA, Haughton VM, William AL - Diagnosis of herniated lumbar disk with computed tomography. N Fingl J Med 301:1J66, 1979.

4. Norlén G - On the value of the neurological symptoms in sciatica for the localization of a lumbar disc herniation: a contribution to the problem of the surgical treatment of sciatica. Acta Chir Scand 91 (Suppl) 95), 1944.

5. Roth-Vargas AA, Balbo RJ, Rossitti SL, Oliveira MA, Sperlescu A, Zulani AR - Acometimento monorradicular neoplásico: diagnóstico diferencial com as discopatias lombares. Tema oficial. XVII Congresso Brasileiro de Neurocirurgia, Brasilia, 17-22 de setembro de 1988 .

6. Schweller OG, Sperlescu A, Balbo R.T, Rossitti SL - Compressåo mielorradicular por cisticercose. Rev Bras Reumatol 28:203, 1988. 